\title{
LA GUERRA EN LA ESCUELA. TEXTOS DE LECTURA Y CELEBRACIONES ESCOLARES EN EL PARAGUAY DE FINES DEL XIX E INICIOS DEL XX
}

\author{
The war at school. Textbooks and school celebrations in Paraguay in the late \\ nineteenth and early twentieth century
}

Ignacio Telesca*

\section{Resumen}

El presente texto ${ }^{* *}$ intenta comprender de qué manera la escuela primaria reflejaba la memoria sobre la guerra que se venía construyendo en el Paraguay de fines de siglo XIX. Los documentos analizados fueron los textos escolares utilizados así como las celebraciones escolares. Estas últimas, al igual que los relatos de los docentes, mediadas por las Revistas Escolares oficiales. Se concluye que no existió una uniformidad en la manera de abordar el tema de la guerra y que ya a principios del siglo XX se levantaron voces preocupadas por la dirección "patriotera" que iban adquiriendo las celebraciones de las fiestas patrias.

$<$ Paraguay $><$ Educación $><$ Guerra de la Triple Alianza $><$ Textos escolares $><$ Celebraciones escolares $>$

\section{Abstract}

This paper aims to understand how primary school reflected the memory of the war that had been built in the turn of the nineteenth century in Paraguay. The documents analyzed were textbooks and school celebrations. The latter, like the narrations of the teachers, were mediated by official school journals. It is concluded that there was no uniformity on the approach to the theme of war and already in the early twentieth century concerned voices arose about the "patriotera" direction that patriotic celebrations of national holidays were gaining.

$$
<\text { Paraguay }><\text { Education }><\text { Triple Alliance War }><\text { School texts }><\text { School Celebrations }>
$$

Recibido: 12/09/2015 // Aceptado: 13/11/2015

\footnotetext{
* Universidad Nacional de Formosa. CONICET. itelesca@hotmail.com

** Quiero agradecer al personal de la Biblioteca Nacional en Paraguay todo el apoyo brindado.
} 
Telesca. La guerra en la escuela. Textos de lectura y celebraciones escolares en el Paraguay de fines del XIX...

Liliana Brezzo, analizando los testimonios de los sobrevivientes de la guerra recogidos por Estanislao Zeballos, afirma “..., a la vista de estos discursos provenientes de los actores paraguayos, que en las últimas décadas del XIX la memoria sobre la guerra, en Paraguay, no era unánime" (Brezzo, 2015: 23). ${ }^{1}$

No era unánime por variadas razones, partiendo del hecho que Asunción, y el Paraguay, estuvo ocupada por las fuerzas aliadas hasta 1876 (Villa Occidental con tropas argentinas, hasta 1878). Al mismo tiempo, al haber sido para la sociedad paraguaya una guerra total (Capdevila, 2007), la experiencia de los sobrevivientes y de los veteranos en particular, también fue múltiple (Gómez Florentín, 2013). La visión de los veteranos se hace aún más heterogénea si tenemos en cuenta que bajo esta categoría también estaban incluidos aquellos que habían combatido bajo la bandera de la Legión Paraguaya (que formaba parte del ejército de la Triple Alianza).

Que la memoria fuese heterogénea no significa que estuviese ausente. Gómez Florentín precisamente llama la atención cómo tras la visita de Estanislao Zeballos a fines de la década de 1880 se dio vigor a "peregrinaciones de la memoria [que] con el tiempo se transformarían en aventuras turísticas que recorrían los lugares de las batallas al paso de las personas que vivieron o recordaban lo sucedido" (Gómez Florentín, 2013: 36).

Puede ser que no haya sido exclusivamente debido a los requerimientos documentales del intelectual argentino el que se hayan iniciado estas peregrinaciones. El mismo Zeballos al llegar al lugar donde se había desarrollado la sangrienta batalla de Tuyutí exclamaba que:

“...se presentó ante mi alma el espectáculo más conmovedor, solemne y doloroso que recuerdo haber presenciado. Dos leguas de cruces y sobre esas cruces sudarios blancos flotaban al viento en esa pavorosa soledad. Eran tiernísimos testimonios que movieron lágrimas en mis ojos fuertes, del amor de las mujeres paraguayas, que todos los años, el 24 de mayo, llegan de los ámbitos de la república en peregrinación enternecedora, a coronar las finas telas bordadas por sus cariñosas manos, los osarios de los muertos quemados, donde yacen cenizas de los que animó su propia sangre, en montones anónimos" (citado por Brezzo, 2015: 9). ${ }^{2}$

\footnotetext{
${ }^{1}$ Estanislao Zeballos, político e intelectual argentino nacido en Rosario en 1854, conoció el Paraguay siendo adolescente, en 1869, y quedó tan impresionado por lo que experimentó que decidió "conocer sus causas, averiguar sus resultados y ponderar sus responsabilidades para los dos pueblos" (citado por Brezzo, 2009-2010: 218).

2 En 1888 el senador Juan G. González propuso convertir a Humaitá en santuario nacional "de esta manera las futuras generaciones de jóvenes verán que sus padres defendieron su territorio palmo a palmo; desde Itapirú hasta Cerro Corá debe llenarse de monumentos que recuerden el heroísmo del pueblo paraguayo" (citado por Warren, 2010: 190-191).
} 
El proceso de memorialización cobró un giro importante hacia fines del siglo XIX y principios del siguiente. Varias fueron las razones que coadyuvaron a que se produjese dicho viraje: una nueva generación de intelectuales formados en el Paraguay posbélico ya ocupaba posiciones políticas de relieve; en 1895 se había formado el Instituto Paraguayo ${ }^{3}$ y al año siguiente difundía su Revista del Instituto; el 16 de agosto de 1899, tras un lustro de debates, se aprobó en el Congreso la ley que garantizaba una pensión monetaria a los veteranos de la guerra; y entre 1902 y 1903 se desarrolló el célebre debate entre Cecilio Báez y Juan E. O’Leary sobre cómo comprender la historia del Paraguay y en particular la Guerra contra la Triple Alianza (Brezzo, 2008).

Es en este contexto en que queremos preguntarnos sobre cómo la educación primaria se hacía, o no, eco de esta coyuntura; de qué manera estos debates se reflejaban, ya sea en los textos de lectura, ya sea en las clases o mismo en las revistas escolares.

Concepción Pérez, alumna maestra del tercer año normal de Asunción, realizó en 1902 una clase modelo frente a sus compañeras sobre la historia patria, clase pensada para alumnos de segundo grado. Antes de realizar la clase disertó sobre el tema en cuestión. La Revista Escolar, órgano del Consejo Nacional de Educación, en el número de julio de 1902 recogió dicha disertación.

Tras un breve recorrido por la historia patria, donde narraba secuencialmente los acontecimientos, Concepción Pérez, llega a lo referente a la Guerra contra la Triple Alianza, y pareciendo hacer una pausa, cambia la densidad en su relato.

"Pero viene por fin la historia (bien triste por cierto) del Paraguay, la de aquella penosa agonía de cinco años, que ha sepultado en el olvido a nuestros padres, parientes y amigos; en que muchas madres arrastrasen la más negra desesperación por nuestros desiertos; pero no hablemos más de aquel pasado tan triste porque tal vez emocionados vuestros corazones con el mío, no cumplamos debidamente nuestro deber" (Revista Escolar, $\mathrm{N}^{\circ}$ 14-15, 1902: 177).

¿Qué es lo que la maestra Concepción Pérez no quería dialogar con su colegas? ¿Por qué no seguir conversando sobre un tema tan central a la historia patria, tópico de la disertación? ¿Qué imaginario sobre la guerra compartían las alumnas de la Escuela Normal, futuras docentes en las escuelas primarias? ¿Cuál era el imaginario compartido por la dirección o el hegemónico entre las autoridades educativas del Paraguay de principios de siglo XX?

\footnotetext{
3 Previo a la conformación del Instituto se había creado el Ateneo Paraguayo en 1883 el cual duró hasta 1889, vísperas de la creación de la Universidad Nacional. Heredero del Ateneo, sus integrantes conformaron el Instituto Paraguayo, en 1895. Agrupaba a miembros de la joven intelectualidad paraguaya, recién egresados de la Universidad con sus mismos profesores en un centro donde se cultivaba la música, las artes, la esgrima, y el aprendizaje de las lenguas. En su revista aparecida al año siguiente se publicaron los escritos más importantes de carácter histórico. Cfr. Amaral, 2003 y el primer número de la Revista del Instituto de 1896 donde su primer artículo se titula "Nuestros Propósitos".
} 
Telesca. La guerra en la escuela. Textos de lectura y celebraciones escolares en el Paraguay de fines del XIX...

Este texto quiere profundizar precisamente en la construcción de la historia de la Guerra contra la Triple Alianza que se fue realizando desde la institución educativa tras la misma guerra. Para tal fin nos hemos de centrar no sólo en los textos escolares utilizados en las aulas, sino también en las publicaciones oficiales y en los discursos pronunciados durante las celebraciones de las fechas patrias. No sólo saber qué se difundía y se leía, sino intentar asir, en la manera de lo posible, la forma en que fueron leídos dichos textos.

Los textos escolares utilizados y las revistas oficiales de educación por un lado; los registros de las celebraciones patrias y los discursos que allí se pronunciaban por el otro. Esta puesta en diálogo de las fuentes nos puede ayudar a comprender si el discurso de los textos (y de cuáles textos) circulaba directamente, mediaba el docente y/o no llegaba.

\section{I}

Apenas concluida la guerra, las mismas autoridades comenzaron a 'escribir la historia' a través de las leyes y los decretos. El 20 de julio de 1871 se aprueba una ley que recupera un decreto del Triunvirato del 17 de agosto de 1869, por la cual se declara fuera de la ley al "desnaturalizado paraguayo Francisco Solano López... y para siempre arrojado del suelo paraguayo como asesino de su patria y enemigo del género humano" (Registro Oficial, 1887: 221). ${ }^{4}$

Si bien un decreto de este tenor tenía cierto sentido en 1869 cuando el mariscal López estaba aún vivo, en pos de deslegitimar todo posible acto suyo de gobierno; ya en 1871, muerto López, se descubre una intención extra, que intenta deslegitimar una posible imagen heroica de López y su modelo de estado. ${ }^{5}$

No se descarta además la mano de las fuerzas de ocupación en este tipo de acto, como en el decreto del 24 de mayo de 1870 en que se declaraba al 25 de mayo (feriado argentino) como feriado en la República del Paraguay; acto reparador, según el decreto, de "falta tan grave y criminal" (Registro Oficial, 1887a: 83; cfr. Caballero Campos, 2013).

Al mismo tiempo, las imágenes que se proyectaban en ciertos textos escolares del Río de la Plata no eran las más halagüeñas para el Paraguay, mucho menos para Francisco Solano López.

La intelectual y pedagoga argentina Juana Manso (1819-1875), en su Compendio de la Historia de las Provincias Unidas del Río de la Plata desde su descubrimiento hasta el año 1874, no dudaba en afirmar que "la Confederación nunca debió reconocer

\footnotetext{
${ }^{4}$ El 15 de agosto de 1869 asumió el gobierno del Paraguay un Triunvirato con el apoyo de las fuerzas aliadas. En esos momentos se puede afirmar que existían dos gobiernos ya que Francisco Solano López continuaba siendo presidente del Paraguay aunque su autoridad se extendía sólo a las fuerzas que lo acompañaban. El Triunvirato continuó hasta el 15 de agosto de 1870 en que se iniciaron las sesiones de la Convención Constituyente (López había sido muerto el 1 de marzo de 1870).

5 Entre los considerando de la Ley de 1871 se insistía "Que la presencia de Francisco Solano López en el suelo paraguayo es un sangriento sarcasmo a la civilización y patriotismo de los paraguayos" (Registro Oficial, 1887: 221).
} 
la independencia del Paraguay" (Manso, 1875: 179-180) y que las intenciones de López eran conquistar Corrientes, el Mato Grosso, el Chaco y las Misiones argentinas, ensanchando así el territorio paraguayo, que "le permitiesen erigir éste en imperio y coronarse él emperador" (Manso, 1875: 180).

Del mismo tenor es la obra de Antonino Luna, ex catedrático de historia universal del Colegio Nacional del Uruguay, Historia de la República Argentina y de las del Paraguay y Banda Oriental desde su descubrimiento hasta nuestros días para el uso de las escuelas, publicado en Buenos Aires por la Imprenta de Pablo E. Coni en 1878. Con gran capacidad de síntesis, resume en una página la historia que va desde la independencia hasta la fecha de publicación de la obra. ${ }^{6}$

Insiste en el estereotipo del pueblo paraguayo ignorante y atrasado que soporta la tiranía de los déspotas. ${ }^{7}$ Sus salvadores, según Luna, fueron los ejércitos aliados quienes:

\begin{abstract}
"despedazando las cadenas que oprimieron al pueblo paraguayo durante tantos años, presenciaron su exterminio y recibieron el último aliento del monstruo Solano López, quien, después de haber sacrificado a los heroicos hijos del Paraguay, en aras de la propagación del terror y de los atentados internacionales, murió cubierto de lodo, como el más digno manto funerario de los Calígulas americanos" (Luna, 1878: 114).
\end{abstract}

Era este el ambiente que se vivía en el Paraguay y en la región en los primeros años de la posguerra sea desde el mismo gobierno o desde los textos escolares extranjeros. Tomar en cuenta estos textos escolares argentinos nos resulta también importante teniendo en cuenta que muchos jóvenes del Paraguay iban a estudiar al vecino país.

\title{
II
}

Si bien desde la nueva Constitución jurada en 1870 se estipulaba que la educación primaria sería "obligatoria y de atención preferente del gobierno", no será hasta 1881 que se escriba el primer Reglamento General para las Escuelas Públicas.

En dicho año, el Estado creó nueve escuelas de primera clase, diez de segunda y cuarenta y cuatro de tercera clase, estableciendo la ubicación de cada una de ellas y la dotación mensual que le correspondía, 50, 20 y 15 pesos fuerte respectivamente (Registro Oficial, 1887b: 794-796). ${ }^{8}$

\footnotetext{
${ }^{6}$ Confrontar el trabajo de Sansón Corbo, 2011 para una reflexión en profundidad sobre la visión rioplatense de la historia del Paraguay.

7 Para un análisis de estos estereotipos en la región ver Capdevila, 2007.

${ }^{8}$ En donde se instalen las escuelas de primera y segunda clase, las Juntas Administrativas debían aportar la misma suma.
} 
Telesca. La guerra en la escuela. Textos de lectura y celebraciones escolares en el Paraguay de fines del XIX...

La enseñanza primaria se dividía en tres grados, que sólo se dictaban en las escuelas de primera clase (ubicadas en las villas importantes como Concepción, Villa Rica o Pilar), en las de segunda solo dos (localidades como Carapeguá o Caazapá), y en las de tercera solamente el primer grado (en los pueblos de Ajos, Capiatá, en los de Misiones, etc.) (Registro Oficial, 1887b: 799).

También el reglamento estipulaba los contenidos a ser dictados en cada grado. La "Historia nacional" se enseñaría desde el primer grado, junto con las nociones de escritura, lectura y aritmética. ${ }^{9}$

Lo importante es tomar conciencia que pasada una década de la guerra la educación primaria completa llegaba aún a muy pocas poblaciones.

El Reglamento General para las Escuelas Públicas señalaba de igual manera como una de sus atribuciones la de fijar los textos de enseñanza a ser utilizados. Esto último no se llevó cabo.

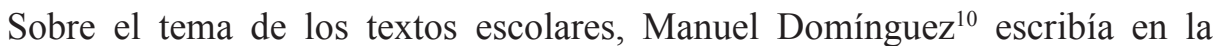
Revista del Instituto en 1897: "No hace 15 años que el catecismo del padre Astete, con sus cuatro infiernos en el centro de la tierra, era el libro clásico de nuestras escuelas. Nosotros le aprendimos de memoria ¡setenta años después de nuestra emancipación política!" (Domínguez, 1897: 233, resaltado en el original). ${ }^{11}$

La primera vez que el Estado nacional invirtió en la compra de textos escolares fue en 1889, "la que fue encomendada al cónsul en Buenos Aires, don Federico Alonso, quien en marzo 89 envió una importante remesa de 25 cajones cuyo contenido fue distribuido entre todas las escuelas nacionales", según se reseña en un artículo aparecido en la Revista Escolar de 1901. (Lelio, 1901: 201).

En la primera revista que editó el Consejo Superior de Educación, La Escuela Moderna, del año 1889, se detalla el contenido de dichos cajones: entre otros, 3.000 ejemplares de El Lector Americano 1, 2.000 del Lector Americano 2, y 1.000 del Lector Americano 3 además de 5.000 ejemplares del Nuevo Libro Primario de los Niños de la editorial Appleton; otros 2.000 de las Lecturas Prácticas de Calixto Oyuela. ${ }^{12}$

\footnotetext{
${ }^{9}$ Las escuelas graduadas recién se implementaron en la década siguiente, pero incluso a principios del siglo XX la mayoría de las escuelas eran las, así llamadas, elementales. Según el Reglamento de 1881, en el primer grado se enseñaban: lectura, escritura, aritmética, gramática, dictado, geografía, historia nacional, doctrina cristiana, moral y urbanidad e instrucción cívica. Estas materias se mantenían en los otros dos grados, y en el tercero se añadía además, entre otras, historia antigua, sagrada y del continente americano. Como método se prohibía "toda enseñanza empírica que esté fundada exclusivamente en la memoria" (Decretos, 1882: 9).

${ }^{10}$ Intelectual paraguayo nacido en 1868, egresado tanto del Colegio Nacional de Asunción como de la Universidad Nacional, llegó a ser vicepresidente de la República entre 1902 y 1904. Fue diputado y Ministro de Relaciones Exteriores, Director del Archivo Nacional y del Colegio Nacional además de docente universitario. Falleció en 1935. Ver Amaral, 2003 y Telesca, 2010.

${ }^{11}$ El Catecismo de la Doctrina Cristiana fue compuesto por el jesuita español Gaspar Astete en el siglo XVI y junto con el de su compañero de orden, Jerónimo Martínez de Ripalda, fueron ampliamente utilizados tanto en España como en América desde ese mismo siglo hasta entrado el siglo XX.

${ }^{12}$ El Consejo Superior de Educación se creó por Ley el 24 de octubre de 1887 (Registro Oficial, 1991: 131137). Los textos de El lector americano, curso gradual de lecturas, fue compuesto por Abelardo Núñez.
} 
Todo este material fue adquirido en la casa Estrada de Buenos Aires y formaban parte también del Catálogo de las obras de texto aprobadas por el Consejo Nacional de Educación argentino, entre el que también estaba el Compendio de Historia de Juana Manso que citábamos al inicio (Cfr. El Monitor, n 170, 1889).

El Consejo, al recibir el material de Buenos Aires, comenzó a repartirlos tanto en la Capital como en la campaña. No se especifica el criterio, pero en la Capital, por ejemplo, se reparten 70 ejemplares de cada uno de los tres Lector Americano y 40 ejemplares del libro de Terán y Gamba cuando en dicho distrito existían 13 escuelas públicas con 36 maestros y 1.263 alumnos (La Escuela Moderna, 109-112).

Es la primera vez que una publicación oficial, de las que hemos revisado, menciona un texto publicado en Paraguay, el Compendio de Geografia e Historia del Paraguay por Leopoldo Gómez de Terán y Próspero Pereira Gamba, publicado por primera vez en 1879 por la Imprenta de La Reforma. ${ }^{13}$

Gómez de Terán, ingeniero napolitano, y Pereira Gamba, abogado colombiano, ambos residentes en Paraguay en esos años, redactaron esta obra pensando en Colegio Nacional en Asunción que se había creado en 1877. De hecho, el texto no se adecua a un alumno de escuela primaria.

Aunque no se especifica, se puede intuir que la distribución de este texto estaba destinada a los docentes y no a los alumnos, y seguramente habrá influido que el mismo Gómez de Terán se desempeñaba como Director de Escuelas Municipales de Asunción.

La obra fue sometida al estudio de los entendidos, y el mismo José Falcón ${ }^{14}$ dio su veredicto positivo aclarando que "aun cuando se pudiera considerar su deficiencia como compendio, las nociones que contienen son verdaderas, según el estudio que tengo hecho y los conocimiento que me han proporcionado los varios documentos del archivo nacional" (Terán y Gamba, 1879: 4).

Es importante señalar que a pesar de los textos escolares que circulaban por la región, y que los autores ciertamente conocerían, no adoptaron una actitud recriminatoria contra Francisco Solano López quien veía, según Terán y Gamba, “en el mantenimiento de la independencia del Estado Oriental una garantía para la seguridad y prosperidad del Paraguay" (Terán y Gamba, 1879: 160). Luego realizaban un somero racconto de las batallas más importantes destacando sí la figura heroica de Bernardino Caballero, quien era, en momentos de la redacción del libro, el hombre fuerte del Paraguay (Terán y Gamba, 1879: 165). ${ }^{15}$

Existen diversas ediciones por país. Para un estudio de Abelardo Núñez, ver Ceballos, 2008.

${ }^{13}$ El texto será conocido como "el Terán y Gamba", así como se expresa en La escuela moderna.

${ }^{14}$ José Falcón, fue el organizador del Archivo Nacional previo a la Guerra y tras ella fue el primer director del mismo Archivo, además ocupó diversos puestos administrativos de importancia. Cfr. Falcón, J. 2006. Escritos Históricos, Asunción: Servilibro. La edición y los estudios preliminares corresponden a Thomas Whigham y a Ricardo Scavone Yegros.

${ }^{15}$ Bernardino Caballero, nacido en 1839, llegó a General durante la Guerra contra la Triple Alianza y acompaño a Francisco Solano López hasta las últimas batallas. Tomado prisionero fue conducido a Río de Janeiro. Regreso en 1872 y comenzó a ocupar puestos en el Poder Ejecutivo hasta que llegó a la presidencia, primero provisional en 1880 y luego constitucional de 1882 a 1886 . Fue uno de los 
Telesca. La guerra en la escuela. Textos de lectura y celebraciones escolares en el Paraguay de fines del XIX...

El Mariscal López pasaba a convertirse en tirano recién tras los tribunales de sangre. ${ }^{16}$ Si bien no presenta una imagen benévola de López, se mira al Paraguay y a la guerra con una simpatía mayor que algunos de los textos que circulaban en el Río de la Plata. ${ }^{17}$

El Compendio de Terán y Gamba, como también señala José Falcón, fue la primera versión narrada de la historia del Paraguay tras la guerra contra la Triple Alianza y se convirtió en el libro de enseñanza no sólo del Colegio Nacional sino de todas las escuelas del país. Para 1890 el texto iba por su duodécima edición, a razón -en promediode una edición por año con una tirada total no menor de diez mil copias. Sólo en el año 1889 se repartieron a las escuelas de todo el país 1.788 ejemplares. En otras palabras, a decir por los números, esta versión de la historia del Paraguay estaba ampliamente difundida en las décadas de los ' 80 y ' 90 en los medios educativos paraguayos.

No fue ésta la única obra que intentó responder a los programas escolares. El profesor Enrique Alliot publicó en Asunción su Elementos de Historia y Geografía en 1884 , editado por la imprenta de La Democracia. ${ }^{18}$ De las once páginas que destina a la "historia particular del Paraguay", seis se refieren al Mariscal López y la guerra, adoptando un tono más severo y condenatorio que el de Terán y Gamba, aunque de esta última obra extrae los párrafos que se refieren a Bernardino Caballero (cf. Alliot, 1884: 11).

En la misma imprenta se publicó en 1887 la Pequeña Geografía (para los niños de la Escuela de Arroyos y Esteros), obra del sacerdote paraguayo Fidel Maíz (18281920). ${ }^{19}$ Este texto, si bien es de geografía, es importante porque se propone responder a los manuales que circulaban en las escuelas. Si bien no cita a ninguno en especial, se pregunta...

"Es por eso que me lastimo al ver que nuestros textos, aquellos cabalmente que deberían despertar en los niños los sentimientos de amor a la patria, sean tomados del extranjero...

¿Cómo nutrir, pero ni despertar estos sentimientos en los tiernos corazones de nuestros educandos por medio de textos de Geografía y de Historia, en que se nos pinta con colores tan sombríos, en que se nos trata de autómatas paraguayos, en

fundadores de la Asociación Nacional Republicana en 1887.

${ }^{16}$ Proceso judicial llevado a cabo en marzo de 1868 en San Fernando, sobre el río Tebicuary a 10 kilómetros de su desembocadura en el río Paraguay. Se acusó a una serie de importantes personalidades, entre ellas el obispo del Paraguay y familiares de Francisco Solano López, de conspirar contra la vida del Mariscal. En la Tabla de Sangre se registra un total de 605 fusilamientos de personas acusadas de traición.

${ }^{17}$ Para una compresión acabada de cómo los textos argentinos y uruguayos se referían a la historia del Paraguay, ver Sansón Corbo, 2011.

${ }^{18}$ El texto fue dedicado "Al Excmo. Sr. Don José S. Decoud, Secretario de Estado y Ministro de Relaciones Exteriores"

${ }^{19}$ Para profundizar sobre esta figura polémica ver Fidel Maíz, Etapas de mi vida, Asunción: El Lector, 1996 con un estudio documental de Carlos Heyn Schupp. Este mismo historiador ha editado los tres tomos de los Escritos del padre Fidel Maiz, 2010-2011, Asunción: Academia Paraguaya de la Historia para el primer tomo y CEADUC para los dos siguientes. 
que no se nos reserva ni siquiera el heroísmo del valor y del patriotismo, y se nos enrostra siempre la culpa de haber tenido tiranos, como si ellos no los hubieran tenido también?” (Maíz, 1890: VIII)

Esta cita nos pone sobre alerta que la narración histórica del pasado reciente estaba siendo cuestionada en ciertos ámbitos. Fidel Maíz era al mismo tiempo una figura de gran importancia tanto en los círculos intelectuales del Paraguay como por su participación durante la guerra como capellán de López y responsable de las acusaciones religiosas contra el obispo Palacios.

Sin embargo, el Consejo Superior de Educación, como ya vimos, en 1889 seguía distribuyendo masivamente la obra de Terán y Gamba y ninguna otra de publicación nacional. ${ }^{20}$

En la década del 90 se experimentarán cambios en el ambiente intelectual paraguayo, de la mano de la fundación de la Universidad Nacional de Asunción a fines de 1889. Los primeros abogados en graduarse, entre los que estaba Cecilio Báez (18621941), lo hicieron en 1893. Esta nueva época intelectual se ve reflejada con la creación, en 1895, del Instituto Paraguayo, y la publicación de su Revista del Instituto al año siguiente.

Entre los discursos de apertura de dicho Instituto encontramos el de Cecilio Báez quien recuerda que "El Paraguay ha atravesado una época de oscurantismo y esterilidad. El buitre de la tiranía batió sobre él sus alas y el espíritu plegó las suyas, como atacado de mortal deliquio" (Revista del Instituto, 1: 10).

En los siguientes cuatro números publicará la Revista las "Cartas polémicas" entre Juan Carlos Gómez y Bartolomé Mitre que habían aparecido en La Tribuna de Buenos Aires en 1869, y que pertenecían al archivo de Enrique Solano López, hijo del Mariscal. ${ }^{21}$ El número 6, de marzo de 1897, está dedicado exclusivamente a brindar diferentes informes sobre lo acontecido el primero de marzo de 1870, incluido un mapa de Cerro Corá. Resulta llamativo que desde el segundo número de la Revista el tema de la guerra estuvo presente entrega tras entrega por más de un año.

Figura importante durante estos años será Enrique Solano López Lynch, quien se desempeñó como Superintendente de Escuelas desde 1894 hasta 1898. Vimos que aportaba documentación para ser publicada, y además durante su gestión se publicó el libro de Manuel de Mendoza, El Lector Paraguayo. ${ }^{22}$

Ésta ya era una obra destinada a la escuela primaria con sesenta lecturas de las cuales treinta y cinco son de carácter histórico y catorce de ellas se refieren

\footnotetext{
${ }^{20}$ De la primera edición de la obra de Fidel Maíz de 1887 se compraron 1000 ejemplares por el Ministerio de Instrucción Pública, sin embargo no parece que hayan sido repartidos, de acuerdo al informe de 1889 .

${ }^{21}$ Enrique Solano López, hijo del Mariscal, había regresado al Paraguay en 1888 (Warren, 2101: 187-193).

${ }^{22}$ Vimos ya que en la década anterior se habían comprado los tres tomos de El Lector Americano de Abelardo Núñez. Manuel de Mendoza era de origen español, se desempañaba como Inspector de Escuela desde 1889 y siguió ligado a la docencia por las siguientes décadas.
} 
Telesca. La guerra en la escuela. Textos de lectura y celebraciones escolares en el Paraguay de fines del XIX...

específicamente a personajes o batallas de la guerra. En éstas se destaca antes que nada el heroísmo y patriotismo de la población que no luchó bajo el temor de un tirano, sino por la independencia de su territorio: "Mártires de lealtad que, fieles a su jefe, le acompañaron hasta la muerte y perecieron sin rendirse" (Mendoza, 1896: 111); y del Mariscal López, dirá, “sucumbió espada en mano,... muriendo intrépido y heroico al lado de sus últimos soldados" (Mendoza, 1896: 113). "Jamás", se refiere en otra lectura, "se borrará de la memoria del pueblo paraguayo el nombre del Presidente Francisco Solano López” (Mendoza, 1896: 167).

Éste pareciera ser la finalidad del texto. En su primera lectura, llamada precisamente "La lectura" le avisa Mendoza a los estudiantes que "leyendo conocerá la relación heroica de la historia patria, legado de honor, depósito sagrado que es preciso conservar incólume" (Ídem: 6).

Ningún otro texto, ni siquiera artículo periodístico había osado rescatar la figura del Mariscal López como lo hiciera Manuel de Mendoza, pero no encontramos, sin embargo, que haya tenido otra edición además de la de 1896.

Ese mismo año de 1896 Blas Garay ${ }^{23}$ publicaba desde Madrid su Compendio Elemental de Historia del Paraguay. ${ }^{24}$ El estilo es muy similar al de Terán y Gamba y en ciertos pasajes pareciera que lo siguió casi tal cual, como por ejemplo cuando se refiere a las expectativas frustradas tras la elección de Francisco Solano López como presidente (Cfr. Terán, 1878: 158 con Garay, 1896: 272).

En esta primera obra, Blas Garay presenta una visión del gobierno de López y la guerra con luces y sombras. Por un lado afirma que la toma del Marques de Olinda fue una agresión injustificada, pero a renglón seguido sostiene que el pueblo "recibió con júbilo la noticia de que la guerra estaba declarada de hecho", y concluye el párrafo: "López marchaba, pues, en este punto, de acuerdo con él, y este entusiasmo de los primeros momentos no decayó nunca" (Garay, 1896: 275). ${ }^{25}$

Reniega de las acusaciones "dictadas por tribunales incapaces de oponerse a las venganzas del sanguinario presidente. En estos procesos eran generalmente fiscales el P. Fidel Maíz, lo coroneles Crisóstomo Centurión y Aveiro, y otros" (Garay, 1896: 294) pero al mismo tiempo sostiene que "Ningún pueblo rayó más alto en el heroísmo con que defendió el suelo de la patria; ninguno llevó a tan extrema abnegación el sacrificio por la integridad del territorio" (Garay, 1896: 295-296).

${ }^{23}$ Garay nació en Asunción en 1873, fue alumno del Colegio Nacional y en 1893 ingresó en la Universidad Nacional, en la facultad de Derecho y Ciencias Sociales. Tras una carrera intensa, egresa con el título de doctor a principios de 1896.

${ }^{24}$ Formaba parte del proyecto editorial asunceno de Agustín Uribe. La primera obra de la colección Biblioteca Paraguaya fue la Descripción e historia del Paraguay y del Río de la Plata por Félix de Azara, en dos tomos.

${ }^{25}$ La toma del buque brasileño Marques de Olinda, el 12 de noviembre de 1864, cuando remontaba el río Paraguay es considerada como el desencadenante de la guerra. 
Al igual que en Terán y Gamba, además del pueblo el principal héroe es Bernardino Caballero y dedica un apartado especial al tratado secreto de la Triple Alianza y critica la 'fanfarronería' de Bartolomé Mitre. ${ }^{26}$

Lo que llama la atención es que la historia escrita por Garay concluye el 1 de marzo de 1870, no continúa ningún apartado sobre lo que vino después, como sí ocurría en las obras de Terán y Gamba y de Alliot.

Blas Garay, desde el título mismo, plantea su obra como alternativa a la de Terán y Gamba. En un artículo suyo aparecido en el periódico asunceno La Opinión indica que si bien es consciente de las deficiencia de su libro lo considera "preferible al que actualmente sirve de texto en nuestras escuelas", refiriéndose al de Terán y Gamba. De hecho, plantea en dicho artículo que su obra tiene "un propósito de reparación" (Telesca, 2012: 252). ${ }^{27}$

Sin embargo, la obra de Blas Garay tuvo una acogida tibia de parte de la intelectualidad asuncena e incluso en la Revista del Instituto, aunque reconocen lo novedoso de lo obra, no la encuentran apropiada para ser utilizada en las escuelas, "porque los niños no deben de aprender sino aquello que está demostrado de modo palmario y tiene toda la autoridad de una cosa debidamente juzgada" (Revista del Instituto, 1897: 53).

Al año siguiente, también en Madrid, Garay publicó una versión más resumida de su compendio titulada Breve Resumen de la Historia del Paraguay. Sigue el mismo esquema del anterior, aunque no menciona ni a los fiscales de sangre ni las fanfarronerías de Mitre. Sin embargo, concluye su obra afirmando que "La gloria que por esta resistencia empeñadísima, sobrehumana, corresponde a López, que la dirigió, no está desgraciadamente exenta de las manchas que sobre ella arrojan sus inauditas e innecesarias crueldades" (Garay, 1897: 139).

Aunque en enero de 1898 fue aceptado el Compendio adecuado por las escuelas del país por el mismo superintendente López, no consiguió que el Estado pagase una edición especial aparte de la que ya había publicado por la casa editorial Uribe.

Según un artículo aparecido en el periódico asunceno La Democracia el 24 de marzo de 1898, fue el Ministro argentino en Paraguay quien le pidió al Presidente que se dejasen de lado dichos libros por ser contrarios a la Argentina. También el periódico señala que varios directores de escuelas se opondrían a la adopción de los textos de Garay.

En gran parte esto se debe a la oposición de Francisco Tapia, Director de la Escuela Normal de Maestros en Asunción. Esta última se había fundado en 1896 y Tapia, maestro normal formado en la Escuela de Paraná, Argentina, fue elegido como su director, traído desde Buenos Aires donde se desempañaba como Inspector Provincial.

\footnotetext{
${ }^{26}$ Este Tratado Secreto fue firmado por los representantes de Argentina, Uruguay y Brasil el 1 de mayo de 1865 y se mantuvo secreto hasta que la prensa inglesa lo divulgó al año siguiente. En este tratado, entre otras cuestiones, se definían los límites que se le obligaría a tener al Paraguay con sus vecinos.

${ }^{27}$ En el mismo artículo Blas Garay se refiere, además de lo fines reparadores, que escribió el libro "por consideraciones de un orden más prosaico", donde lo económico no ha de dejarse de lado.
} 
Telesca. La guerra en la escuela. Textos de lectura y celebraciones escolares en el Paraguay de fines del XIX...

En ese mismo año se creó en la capital la Escuela Normal de Maestras cuya dirección le cupo a Adela Speratti, paraguaya formada en la Escuela Normal de Concepción del Uruguay.

Tapia utilizaba como texto de historia nacional el libro de Terán y Gamba y se oponía tanto al de Mendoza como al de Blas Garay (Tapia, 1898). Este último se quejará desde su periódico La Prensa en marzo de 1898 porque los maestros no utilizan los textos adoptados por el Consejo de Educación. El acusado será Tapia quien al utilizar la obre de Terán y Gamba marcaba, según la denuncia, la tendencia en el resto de las escuelas.

De igual modo, en marzo y abril de ese año Francisco Tapia y Blas Garay intercambiarán denuncias aunque sin nombrarse. A principios del año escolar de 1898 se confeccionaron cuadernos con biografías de héroes del Paraguay, entre ellas la de Francisco Solano López. Al enterarse Francisco Tapia mando prohibir el uso de dichos cuadernos en las instituciones escolares a su cargo alegando que se estaba falseando la historia nacional a la par que contraviniendo las leyes (Tapia, 1898).

Blas Garay por su parte a través de su diario, La Prensa, señalaba que "abusos de tal índole no pueden ni deben ser tolerados. No se trata de un tirano, se trata de un gobernante paraguayo... que debe merecer respeto" (23 de marzo de 1898). Hubo marchas en contra de Tapia y solicitadas a favor del mismo.

Éste era el contexto en que cobra ahora todo sentido la disertación en 1902 de Concepción Pérez que citábamos al inicio del texto.

Blas Garay muere en un duelo al año siguiente y Francisco Tapia dejará la dirección de la Escuela Normal; pero el debate sobre la historia nacional no estaba concluido. No se publicarán más textos escolares sino que los de Garay, Mendoza y Terán y Gamba circularán por las diferentes escuelas (en especial los dos primeros), quedando a la iniciativa del docente los contenidos a enseñar. ${ }^{28}$ Así se desprende de la vivencia de César Martín, maestro de San Bernardino.

"Sin buenos textos, [afirma César Martín] sin libros con relaciones históricas, sin cuadros murales que puedan servir de levadura para una buena lección; tan solos "El breve resumen de historia patria" por Garay o "El Lector Paraguayo" por Mendoza, son los únicos libros al alcance de un maestro de campaña; libros insuficientes, teniendo en cuenta que en historia, como en todo, hay que saber mucho para enseñar bien un poco (Revista de Instrucción Primaria, $\mathrm{N}^{\circ} 2$ 2, 1903: 86).

\footnotetext{
${ }^{28}$ En lo que se refiere a libros de historia nacional desde la obra de Garay en 1897 no se publicarán más, sino sólo libros de lecto-escritura como El Nene Paraguayo de Manuel Chaves siguiendo el modelo de la obra $E l$ Nene de Andrés Ferreyra (1865-1928), sin abordar en las lecturas temas relacionados con la historia. La obra del pedagogo argentino publicada por primera vez en 1895 (y con sucesivas ediciones hasta 1959) proponía un sistema gradual de enseñanza de la lecto-escritura.
} 
Se puede percibir que ya para estos años la obra de Terán y Gamba estaba siendo cuestionada en cierto ambiente escolar. De hecho, en la Revista Escolar que era el órgano del Consejo Nacional de Educación, de abril de 1901, Carlos Lelio hace una crítica muy acabada de la historia de Terán y Gamba. Entre otros puntos critica el dejar relegada a la figura del Dr. Francia y el no tomar en consideración ni siquiera los héroes de la guerra. Su conclusión es que

“...mejor sería suprimir tal obra como libro de texto y mientras aparezca una que satisfaga íntegramente las variadas exigencias que debe llenar el interesante estudio de la historia patria, dejar al criterio de los maestros la elección y consulta de los textos y diversas publicaciones que con más fundamentos y con tendencias verdaderamente científicas y morales 'hagan comprender a los niños el origen, carácter y condición de la nación de que forman parte, y los prepare para el ejercicio inteligente de sus deberes y responsabilidades como ciudadano"” (Revista Escolar, 1901: 374).

Sin embargo, a pesar del estudio que podamos realizar alrededor de los textos escolares debemos analizar la llegada de los mismos a los alumnos y de cómo los maestros, cuando estos textos no llegaban, intermediaban entre los textos y los escolares. Del mismo maestro de San Bernardino, César Martín, podemos ver qué uso se hacía también de dichos textos en el aula misma:

“...Acomodándome a las circunstancias que me rodean, sin grabados, sin estampas, sin cuadros que representen los grandes hechos y personajes de la historia nacional, comprendiendo que la enseñanza oral es deficiente, dado que la lengua nacional es la que menos entienden mis alumnos, he adoptado el método siguiente: Elegido el tema arreglado al programa, refresco la memoria con textos a mano. Hecho un resumen escrito, con él me presento a clase. Leído éste, hago preguntas sobre los hechos más importantes, fijándome en aquellos discípulos que menos conocen el castellano, para hacerles hablar, idea primordial que llevo en todas mis lecciones. Concluido este ejercicio, transcribo en la pizarra mi resumen, para que lo copien en cuadernos preparados al efecto. Casi siempre, se repite la lección en la conferencia próxima" (Revista de Instrucción Primaria, 1903: 88).

Este testimonio sumado a muchos otros proveniente del interior del Paraguay deja constancia que más que por uso de los textos escolares por parte de los alumnos, la 
transmisión de la historia estaba fundamentalmente intermediada por los docentes, y la memorización la técnica más utilizada. La maestra Bertilda Shuttleworsk desarrollaba una clase sobre la vida del General José E. Díaz utilizando métodos parecidos al de César Martín: preguntas-respuestas ya dadas y escritura de las mismas; luego repetición (Revista Escolar, 1901: 509-510). ${ }^{29}$

De igual manera queda también de manifiesto en el Informe pasado a la Dirección de Escuelas por el Inspector General Manuel W. Chaves, el 23 de enero de 1901 en lo que se refiere a la enseñanza de la historia:

"En la mayoría de nuestras escuelas considero su enseñanza defectuosa; en los métodos que se emplean no hay deducción de los hechos y acontecimientos, todo se deja librado al esfuerzo nemotécnico del educando. La inspección ha procurado que no se haga tanto uso de los textos para la enseñanza de esta asignatura; más todavía, teniendo en cuenta que nos hace falta, mucha falta, un historiador que presente a cada uno de nuestros personajes con sus vicios y virtudes y trate los hechos y acontecimientos con la imparcialidad y perfección que debe emplear el investigador amante de la verdad y de la justicia..." (Chaves, 1901: 24).

Sin lugar a dudas, el cambio de siglo fue un momento de mucha efervescencia alrededor de la enseñanza de la historia: qué enseñar, cómo enseñar y para qué enseñar. Ramón Indalecio Cardozo, director de la Escuela Graduada de varones de Villarrica, en su conferencia dada en ocasión de la fiesta del 14 de mayo, también se refirió al tema y de una manera muy puntual:

“...para que su enseñanza resulte fecunda hay que seguir el siguiente plan: despertar en los niños el amor al suelo natal, el cariño a sus héroes y veneración a sus mártires, por medio de lecciones que hablen, en conformidad con el estado de desarrollo mental, al sentimiento; y hacer que investiguen el origen y causa de los hechos sociales, que comparen entre sí los sucesos históricos, los personajes que han actuados en ellos con relación al medio social, y sacar las consecuencias ya sean buena o fatales.

Con la primera fase de la enseñanza de la Historia formaremos ciudadanos amantes de la patria, cuyo infortunio o grandeza

${ }^{29}$ El Gral. Díaz era la figura heroica de la guerra; vencedor en la batalla de Curupayty era el personaje más recordado en los discursos y tareas escolares. Estanislao Zeballos se refiera a él como "Este hijo del pueblo, ennoblecido por su valor y cierta altivez de carácter, ante el mismo López, es el héroe popular de la guerra. Los paraguayos le cantan todavía versos compuestos a su memoria" (Brezzo, 2015: 79-80). 
habrale impresionado, porque habremos llevado a la mente infantil por medios concretos, la imagen de la patria idolatrada. Con la otra, investigadores capaces de pensar en serio, cuando grandes, en el porvenir de la patria." (Revista Escolar, 1901: 490).

El aula era el ámbito de acción de los docentes y los contenidos que enseñaban no siempre iban de la mano con las indicaciones del ministerio. Por otro lado, la mayoría de los educadores en esto años no habían sido formados en las escuelas normales y sus influencias no se dejan sentir en todos los rincones de la patria. ${ }^{30}$

Según David Velázquez (2015) hacia 1900 la mayoría del personal docente que enseñaba en las escuelas del Paraguay no había pasado por las Escuelas Normales. Su formación autodidáctica venía acompañada de los textos escolares recibidos desde Asunción y de las diversas Revistas Escolares. Es muy difícil por lo tanto trazar una línea ideológica teniendo en cuenta que en los primeros años posbélicos muchos también eran extranjeros.

El 24 de diciembre de 1900, la directora de la escuela graduada de Villa Rica, Lorenza T. de Vico le escribe al presidente Emilio Aceval sobre su actividad en la escuela: "Aunque muy humilde, mi contingente en la gran obra regeneradora, estoy dispuesta hasta el sacrificio si fuera necesario, consagrar todos mis esfuerzos para contribuir a la realización de los grandes ideales en pro de esta patria tan digna por su heroísmo y cruentos sacrificios." (Revista Escolar, 1900: 170-171)

Cuando se revisan las revistas escolares de la época donde se registran discursos, ponencias o incluso las celebraciones festivas, ${ }^{31}$ la idea de patria, del pueblo heroico, de figuras como el General Díaz aparecen constantemente. Como en el resto de la sociedad, la memoria sobre la guerra tampoco era unánime y se percibe un ligero cambio en las publicaciones con el correr de los años hacia una reivindicación mayor de tal acontecimiento.

Esta falta de unanimidad memorial queda reflejada en las celebraciones del 25 de noviembre en 1889 en las escuelas asuncenas.

Por un lado, Ángel Vázquez, Preceptor de la escuela de niños de la Catedral, tras una semblanza de los héroes de la independencia (sin mencionar, sin embargo, al figura del Dr. Francia) se refería a la guerra y expresaba que "no quiero tratar de enternecer a mis conciudadanos, haciéndole recordar la luctuosa y desastrosa época de

\footnotetext{
${ }^{30}$ El dilema entre los valores cívicos o militares como definidores de espíritu patriótico se puede ver reflejado también en la poesía de Ramón Melgar, "El educacionista y el soldado" aparecida en el número 3 de la Revista Escolar, en enero de 1901 (174-175). Melgar era argentino y la poesía pensada en términos generales y no referentes al Paraguay; sin embargo, que la Revista lo haya incluido nos señala la necesidad que tenía el órgano educativo nacional de hacer hincapié en los valores cívicos.

${ }^{31}$ Las fiestas patrias celebradas en esos años eran el 14 de mayo, independencia, y el 25 de noviembre, jura de la Constitución.
} 
Telesca. La guerra en la escuela. Textos de lectura y celebraciones escolares en el Paraguay de fines del XIX...

nuestros mayores, recuerdo que bien van borrándose de nuestra memoria en presencia de esta nueva época..." (La Escuela Moderna, № 10, 1889: 188).

Por otro lado, también se reproducen en dicha publicación discursos de los alumnos como el de Irene Degásperi quien, delante del Presidente Patricio Escobar (general del Mariscal López durante la guerra), sostenía “que 19 años hace que eminentes patriotas paraguayos juraron nuestra Constitución, sostener nuestra libertad y nuestros derechos o regar con su noble sangre el sagrado suelo de nuestra patria..." (La Escuela Moderna, 1889: 190).

Una década y media más tarde, esta misma falta de unanimidad se refleja en los discursos para las celebraciones de mayo de 1903. La Revista de Instrucción Primaria dedica su número de mayo de dicho año a compartir con sus lectores, que eran otros maestros (se hacían 600 ejemplares), los programas de las diversas celebraciones tanto en la capital como en el interior. Respecto a la guerra y al patriotismo los discursos van de una crítica a la tiranía y a la guerra como el de Jorge Trigo, en Luque, hasta el de Eladio Argüello, en Asunción, donde reclamaba que "A la patria se la debe amar sin reserva, sin reparos ni atenuaciones como se ama a la madre que nos ha brindado con la rica leche de su pecho: por ella se debe amar cuanto ella ame, y aborrecer cuanto aborrezca ella. El hombre verdaderamente patriota debe contener... hasta la voz de su conciencia... ¡El honor de la bandera, la salud de la patria lo piden, lo reclaman, lo exigen todo!" (Revistas de Instrucción Primaria, 1903: 152-153). ${ }^{32}$

Entrados ya en la primera década del siglo XX y tras haberse desarrollado el célebre debate entre Cecilio Báez y Juan E. O'Leary, las fiestas patrias se van convirtiendo en actos que escapan al control de las autoridades educativas. Juan Ramón Dalhquist, ${ }^{33}$ Inspector General de Escuelas presenta al Director General de Escuelas su memoria anual el 31 de enero de 1908 en la que, entre otros ítems, aborda las celebraciones de las fiestas patrias y afirma:

"No puede negarse que con estas fiestas viene introduciéndose abiertamente un patrioterismo funesto, que bien puede llegar a domeñar el corazón de la niñez que se levanta... bien conoce el señor Director a qué clases de abusos suelen prestarse esas fiestas y de consiguiente los resultados suelen ser frecuentemente contraproducentes" (Revista de Instrucción Primaria, 1908: 341; resaltado en el original).

Reconoce el Inspector General lo eficaz de las fiestas para generar cariño en los estudiantes hacia la nacionalidad y los símbolos patrios, pero se queja de "la excesiva

32 Estas ideas ya las expresaba el mismo Fidel Maíz en su Geografía. Allí expresaba que "Las cicatrices de una madre deben cubrirse con respeto y veneración, y por pobre y humillada que haya sido, no se la puede dejar de amar, mucho menos se la puede cambiar por otra, aun cuando ésta sea opulenta y coronada" (Maíz, 1890: VIII).

33 Juan Ramón Dalhquist, nació en Argentina en 1884, hijo de madre paraguaya se radicó en Paraguay desde pequeño. Maestro normal especializado luego en la Escuela Normal de Paraná. 
vulgarización de dichas fiesta" (Ídem). La solución propuesta por Dalhquist no era la de atacar las causas de dicho patrioterismo sino se basó simplemente en reducir el número de fiestas patrias a celebrar.

\section{III}

Si las alarmas que el Inspector Dalhquist levantaba en 1908 eran ciertas, y los hechos posteriores nos mostrarán de qué manera lo eran, ${ }^{34}$ nos queda concluir que en esas décadas el Consejo Nacional de Educación no logró, si ese era su objetivo, unir la idea de amor a la patria a un imaginario cívico.

Si como señala Liliana Brezzo la memoria sobre la guerra no era unánime, tampoco lo era a nivel educativo y esto se aprecia con claridad analizando las revistas escolares de la época, como bien indica Liliana Brezzo, "el pasado no era el mismo para todos" (Brezzo, 2001: 167). En el aula y en los discursos de las fiestas escolares, y siempre estos mediados por las revistas, nos indican que no había una postura decidida de cómo abordar el tópico de la pasada guerra, de cómo relacionar la educación primaria con la construcción de una ciudadanía no marcada por un nacionalismo guerrero.

Para inicios del siglo XX esto queda más en evidencia reflejando las disputas que transcurren más allá de los centros escolares. Lo que la sociedad no había aún resuelto, la escuela tampoco pudo lograrlo.

\section{Referencias bibliográficas}

$\underline{\text { Revistas escolares }}^{35}$

La Escuela Moderna. 1889. Órgano del Consejo Superior de Educación, Asunción.

La Enseñanza. 1899. Periódico quincenal (directores, Manuel W. Chaves y Héctor L. Barrios), Asunción.

El Didascólogo. 1900. Órgano quincenal de Educación (director, Héctor L. Barrios), Asunción, 1900.

Revista Escolar. 1900-1902. Órgano del Consejo Nacional de Educación, Asunción.

Revista de Instrucción Primaria. 1902-1909. Órgano del Consejo Nacional de Educación, Asunción.

\section{Manuales}

Alliot, E. 1884. Elementos de Historia y Geografía. Asunción: La Democracia Garay, B. 1896. Compendio Elemental de Historia del Paraguay. Madrid: Uribe Garay, B. 1897. Breve Resumen de la Historia del Paraguay. Madrid: Uribe,

\footnotetext{
${ }^{34}$ Para un análisis del ‘despertar' nacionalista ver los textos de Brezzo, 2010 y Rivarola, 1993.

${ }^{35}$ La fecha de culminación responde al último número existente en la Biblioteca Nacional, Paraguay.
} 
Luna, A. 1878. Historia de la República Argentina y de las del Paraguay y Banda Oriental desde su descubrimiento hasta nuestros días para el uso de las escuelas. Buenos Aires: Imprenta de Pablo E. Coni.

Maíz, F. 1890. Pequeña geografía (para los niños de la escuela de Arroyos y Esteros). Asunción: edición del autor.

Manso, J. 1875. Compendio de la Historia de las Provincias Unidas del Río de la Plata desde su descubrimiento hasta el año 1874. Buenos Aires: Tipografía del Diario La época, 6 a edición.

Mendoza, M. de. 1896. El lector paraguayo. Asunción: Tallares Nacionales de H. Kraus. Terán Gómez, L. de y Pereira Gamba, P. 1879. Compendio de Geografia e Historia del Paraguay, Asunción: La Reforma (conocido como “Terán y Gamba”).

\section{Textos citados}

Amaral, R. 2003. Escritos Paraguayos, Vol. I. Introducción a la cultura nacional. Asunción: Distribuidora Quevedo.

Brezzo, L. 2001. "E1 Paraguay y la Argentina en los textos escolares: una perspectiva bilateral de las representaciones del otro". Entrepasados, № 20/21, pp. 163-194.

Brezzo, L. 2008. "En el mundo de Ariadna y Penélope: hilos, tejidos y urdimbre del nacimiento de la historia en el Paraguay". En: Báez, Cecilio y Juan E. O’Leary. Polémica sobre la historia del Paraguay, Asunción: Tiempo de Historia, pp. 1163.

Brezzo, L. 2009-2010. "La Guerra de la Triple Alianza en la primera persona de los vencidos: el hallazgo y la incorporación de la Sección Estanislao S. Zeballos del Ministerio de Defensa del Paraguay". Anuario, 22, pp. 217-235.

Brezzo, L. 2010. "Paraguay: reconstrucción, poder político y revoluciones, 1870-1920", Telesca I. (coord.) Historia del Paraguay, Asunción: Taurus, pp. 199-225.

Brezzo, L. (Ed.). 2015. La Guerra del Paraguay en primera persona. Testimonios inéditos. Fondo Estanislao Zeballos. Asunción: Tiempo de Historia.

Caballero Campos, H. 2013. "Fiesta y nación en Paraguay. Las celebraciones de la independencia durante el siglo XIX". Folia Histórica del Nordeste, $\mathrm{N}^{\circ} 21$, pp. 57-74.

Capdevila, L. 2007. Une guerre totale. Paraguay, 1864-1870. Essai d'histoire du temps présent, Rennes: Presses Universitaires de Rennes (hay traducción castellana, 2010, Asunción-Buenos Aires: CEADUC-SB).

Ceballos, A. 2008. "Las empresas editoriales de José Abelardo Núñez en Alemania, 1881-1905". Historia, Vol. 41, № 1, pp. 43-62.

Chaves, M. W. 1901. Informe a la Dirección S. de Escuelas. Asunción: La Tribuna

Decretos sobre reorganización de las escuelas públicas y reglamentación de las mismas (1882). Asunción: Imprenta La Democracia.

Domínguez, M. 1897. "Segunda Conferencia”, Revista del Instituto, N 10, pp. 217-270.

Gómez Florentín, C. 2013. Los veteranos. Asunción: El Lector.

Lelio, C. 1901. "Ojeada retrospectiva”. Revista Escolar, N 4, pp. 201-203. 
Registro Oficial de la República del Paraguay correspondiente a los años 1869-1875. 1887a. Asunción: Editores Fischer y Quell.

Registro Oficial de la República del Paraguay correspondiente a los años 1876-1885. 1887b. Asunción: Editores Fischer y Quell.

Registro Oficial correspondiente al año 1887. 1891. Asunción: Tipografía y Encuadernación de "La República".

Rivarola, M. 1993. La contestación al orden liberal. La crisis del liberalismo en la preguerra del Chaco. Asunción: CDE.

Sansón Corbo, T. 2011. "Identidad y alteridad en los manuales de Historia rioplatenses. Las representaciones de Paraguay". Diálogos, Vol. 13, N 1, pp. 41-63.

Tapia, F. 1898. El tirano Francisco Solano López arrojado de las escuelas. Asunción: Escuela Tipográfica Salesiana.

Telesca, I. 2010. "Paraguay en el centenario: la construcción de la nación mestiza". Historia Mexicana, Vol. LX, ํ1, pp. 137-195.

Telesca, I. 2012. "Blas Garay y el 'Prólogo' a del Techo: inventando el Paraguay". Telesca, I. y Insaurralde, G. (eds.), Melià. Escritos de Homenaje, Asunción, CEPAG, pp. 243-263.

Velázquez Seiferheld, D. 2015. Relaciones entre autoritarismo y educación en el Paraguay 1869-2012. Un análisis histórico. Primer Volumen, 1869-1930. Asunción: SERPAJ.

Warren, H. G. 2010. La reconstrucción del Paraguay, 1874-1904. Asunción: Intercontinental (traducción del original inglés de 1985). 
